\title{
Prostate cancer identification: quantitative analysis of T2-weighted MR images based on a back propagation artificial neural network model
}

\author{
ZHAO Kai ${ }^{1}$, WANG ChengYan ${ }^{2}$, HU Juan ${ }^{1}$, YANG XueDong ${ }^{1}$, WANG He ${ }^{1}$, LI FeiYu ${ }^{1}$, \\ ZHANG XiaoDong ${ }^{1}$, ZHANG Jue ${ }^{2} \&$ WANG XiaoYing ${ }^{1,2 *}$ \\ ${ }^{1}$ Department of Radiology, Peking University First Hospital, Beijing 100034, China; \\ ${ }^{2}$ Academy for Advanced Interdisciplinary Studies, Peking University, Beijing 100871, China
}

Received March 20, 2015; accepted April 23, 2015; published online May 27, 2015

\begin{abstract}
Computer-aided diagnosis (CAD) systems have been proposed to assist radiologists in making diagnostic decisions by providing helpful information. As one of the most important sequences in prostate magnetic resonance imaging (MRI), image features from T2-weighted images (T2WI) were extracted and evaluated for the diagnostic performances by using CAD. We extracted 12 quantitative image features from prostate T2-weighted MR images. The importance of each feature in cancer identification was compared in the peripheral zone (PZ) and central gland (CG), respectively. The performance of the computer-aided diagnosis system supported by an artificial neural network was tested. With computer-aided analysis of T2-weighted images, many characteristic features with different diagnostic capabilities can be extracted. We discovered most of the features $(10 / 12)$ had significant difference $(P<0.01)$ between PCa and non-PCa in the PZ, while only five features (sum average, minimum value, standard deviation, 10th percentile, and entropy) had significant difference in CG. CAD prediction by features from $\mathrm{T} 2 \mathrm{w}$ images can reach high accuracy and specificity while maintaining acceptable sensitivity. The outcome is convictive and helpful in medical diagnosis.
\end{abstract}

prostate cancer, magnetic resonance imaging, T2WI, diagnosis, computer-assisted

Citation: Zhao K, Wang CY, Hu J, Yang XD, Wang H, Li FY, Zhang XD, Zhang J, Wang XY. Prostate cancer identification: quantitative analysis of T2-weighted MR images based on a back propagation artificial neural network model. Sci China Life Sci, 2015, 58: 666-673, doi: $10.1007 / \mathrm{s} 11427-015-4876-6$

Prostate cancer (PCa) is one of the most common malignancies in elderly men worldwide. Its incidence and mortality rate are rising, which is particularly prominent in an aging society [1-4]. MR imaging depicts the anatomy of the prostate with exquisite detail because of its high spatial resolution, superior soft-tissue contrast resolution, and multiplanar capability. It is the most accurate noninvasive method for PCa detection and can also aid local and distant staging [5-8].
T2-weighted images (T2WI) is an essential sequence used in the diagnosis of PCa [9-11]. No matter how many sequences are combined to detect $\mathrm{PCa}, \mathrm{T} 2 \mathrm{WI}$ is always the indispensable one [12,13]. Typical PCa and non-cancer can be differentiated by T2WI. Traditional MR cancer detection mainly relies on the low signal intensity in T2WI, especially for the tumours located at the peripheral zone (PZ), which count for $70 \%-75 \%$ of all PCa [14].

Subjective interpretation of MR images depends on radiologists' experience and expertise, limiting the accuracy of $\mathrm{PCa}$ detection. The inter-observer variability may result in

*Corresponding author (email: cjr.wangxiaoying@ vip.163.com) 
totally different decisions from the same set of images $[15,16]$. Standard and extensive training is needed to improve diagnostic accuracy. However, even an experienced radiologist may still miss some inner characteristics. Computer-aided diagnosis (CAD) [17], in which computer analysis methods are used to help radiologists detect and diagnose, has the potential to address these challenges. Seltzer et al. [18] confirmed that a combined radiologist-computer system substantially improved accuracy of body MR radiologists in the diagnosis of PCa.

Due to the quantitative feature of CAD analysis, it has been developed for breast, lung, and colorectal cancers [19-21], but there has been limited application to PCa, and even rare analysis to T2WI.

The main objective of this study was to extract image features from prostate T2WI and evaluate their diagnostic performances by CAD.

\section{Materials and methods}

\subsection{Study population}

Between December 2008 and January 2010, 71 consecutive patients who were suspected of $\mathrm{PCa}$ in the prostate MR database were selected for the retrospective study. Among them, 35 patients were confirmed to have PCa by biopsy, and the other 36 patients had not been detected of cancer by serial biopsy and long-term follow-up (12 to 59 months, mean 32 months).

Inclusion criteria for the study were as follows: (i) there were complete prostate MR data including T2WI; (ii) the clinical information contained age, total prostate specific antigen (tPSA), free prostate specific antigen/total prostate specific antigen (fPSA/tPSA), ultrasound, and digital rectal examination; (iii) subsequent ultrasound-guided biopsy was performed within 3 months after the MR scan; (iv) all the patients underwent a long-term follow-up. Exclusion criteria were as follows: (i) ultrasound-guided biopsy was performed within 3 months before the MR scan; (ii) the patients were previously diagnosed with $\mathrm{PCa}$ or prostate sarcoma; (iii) there was treatment before the MR scan, such as hormone therapy, radiotherapy, and surgery.

\subsection{MR image data acquisition}

All MR examinations were carried out on a 1.5 Tesla MR scanner (Signa TM; GE Medical Systems, Milwaukee, WI, USA) using a pelvic phased-array coil (GE Medical Systems). All routine prostate MR examinations followed standardised protocols. T2-weighted turbo-spin-echo images were obtained in the axial, sagittal, and coronal planes without fat suppression with TR: $3500 \mathrm{~ms}$; TE: $85 \mathrm{~ms}$; FOV: $240 \mathrm{~mm} \times 240 \mathrm{~mm}$; matrix: $320 \times 256$; slice thickness: $4 \mathrm{~mm}$ with no gap; number of signals acquired: 4 .

\subsection{ROIs selection}

Firstly, regions of interest (ROIs) with $15-20 \mathrm{~mm}^{2}$ within a sextant were manually delineated in the prostate T2WI. ROIs were drawn in the most suspected regions at T2WI by two experienced radiologists ( 7 and 9 years of prostate MR experience) without reference to histopathology. If the region was absolutely normal (no signs of cancer or inflammation), a ROI was put in the mirror position of the contralateral area. The two experienced radiologists also graded the overall score subjectively (5-point scale: 1-definitely benign; 2-probably benign; 3-indeterminate; 4-probably malignant; and 5-definitely malignant) of each ROI according to European Society of Urogenital Radiology (ESUR) prostate guidelines [22] without reference to histopathology.

Then, cancerous ROIs were defined as the ROIs within a sextant that was positive at biopsy, while the non-cancerous ROIs were defined as the ROIs within a sextant that was negative at biopsy, either an inflammation area or a normal area. In total, 238 ROIs were selected from the PZ (146 non-cancerous and 92 cancerous ROIs) and 188 ROIs were selected from the central gland (CG) (136 non-cancerous and 52 cancerous ROIs). The distribution of ROIs selected from the T2WI is summarised in Table 1.

\subsection{Computer-extracted features}

The general data flow diagram of this CAD system can be divided into three main steps (Figure 1): data preparation (images acquisition and annotate MR images), parameters preparation (feature extraction and selection), and prediction (based on artificial neural networks (ANN) classifier) and evaluation (via cross-validation). All were performed with an in-house computer software.

Before analysis, T2WI were standardised to correct for background and nonlinearity of the MR image intensity scale [23]. Quantified features in this study were grouped into three types: general features (mean value, minimum value, standard deviation, coefficient of variation, 10th percentile), features derived from gray-level histogram (skewness, kurtosis), and features derived from co-occurrence matrix (GLCM) (contrast, correlation, energy, homogeneity, entropy) (see Supporting Information). In total, 12 features were extracted from the T2WI. A summary of these features and their descriptions are given in Table 2.

Table 1 The distribution of ROIs in the peripheral zone and central gland ${ }^{\text {a) }}$

\begin{tabular}{cccc}
\hline & Peripheral zone & Central gland & Whole gland \\
\hline PCa & 92 & 52 & 144 \\
Non-PCa & 146 & 136 & 282 \\
Total & 238 & 188 & 426 \\
\hline
\end{tabular}

a) PCa, prostate cancer; ROIs, regions of interest. 


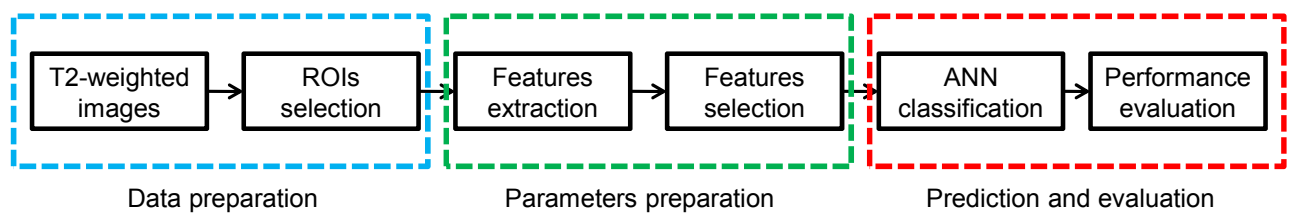

Figure 1 (color online) General data flow diagram of this CAD system.

Table 2 List of features extracted from T2-weighted images

\begin{tabular}{|c|c|c|}
\hline Type & Features & Description \\
\hline \multirow{4}{*}{ General features } & Sum average & Measure of overall image brightness \\
\hline & Minimum value & Measure of the darkest brightness of the image \\
\hline & Standard deviation & Measure of how spread out the gray-level distribution is \\
\hline & $\begin{array}{l}\text { Coefficient of } \\
\text { variation }\end{array}$ & Normalised measure of dispersion of the gray-level distribution \\
\hline \multirow{2}{*}{ Gray-level Histogram } & 10th percentile & Measure of the value below which are $10 \%$ of the cumulative probability values \\
\hline & Kurtosis & Measure of how outlier-prone a distribution is \\
\hline \multirow{4}{*}{ Gray-level co-occurrence matrix [34] } & Contrast & Measure of the local variations in the gray-level co-occurrence matrix. \\
\hline & Correlation & Measure of the joint probability occurrence of the specified pixel pairs \\
\hline & Energy & Measure of the sum of squared elements in the GLCM \\
\hline & Homogeneity & $\begin{array}{l}\text { Measures the closeness of the distribution of elements in the GLCM to the GLCM diago- } \\
\text { nal }\end{array}$ \\
\hline
\end{tabular}

\subsection{Feature selection}

To select the most informative set of features, sequential forward selection (SFS) was performed with an ANN classifier. Prediction error was used as a selection criterion. An SFS algorithm is a bottom-up search procedure which starts from an empty feature set and gradually adds features selected by some evaluation function that minimises the mean square error (MSE). Another goal of feature selection was to preserve the robustness of the classification method and reduce the risk of over-fitting [24].

\subsection{Classifier design}

A devised feed forward ANN structure with three layers (input layer, hidden layer, and output layer) was used as a classifier to identify PCa (Figure 2). The selected features were used as the inputs of the classifier, and the output was the prediction of PCa (between 0 and 1). ROIs in the PZ and CG were trained and tested separately.

In order to improve the characteristics of this classifier, a back-propagation (BP) algorithm was used in the training procedure, and the Levenberg-Marquardt (LM) algorithm was applied as a learning rule. Different transfer functions were tried in the hidden and output layers, the log-arithmicsigmoid (Logsig) was finally selected as the transfer function. The network structure with a hidden layer of 14 neurons and the epoch number of 500 was used. At the end, the network structure that yielded the best classification is given in Table 3.

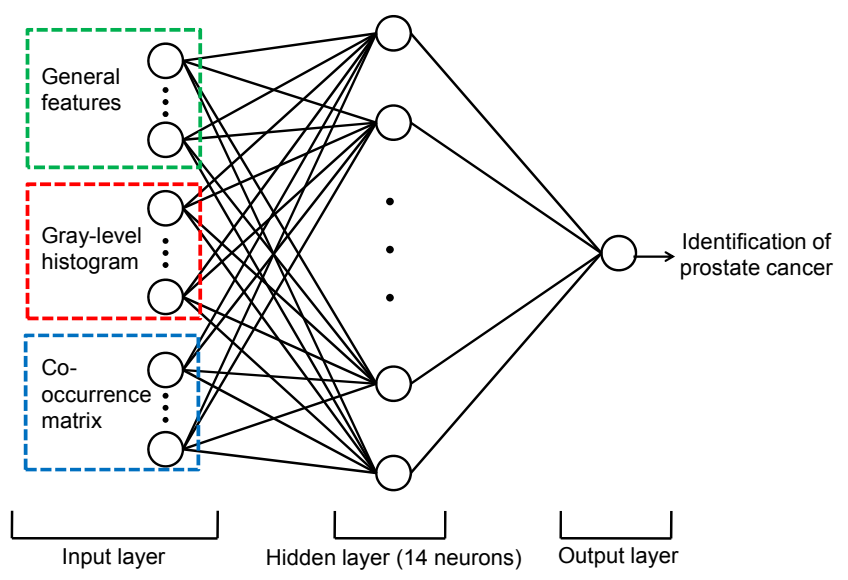

Figure 2 (color online) Schematic diagram of this ANN classifier with three layers (input layer, hidden layer, and output layer). Three kinds of features (general features, texture features derived from gray-level histogram, and co-occurrence matrix) were selected as the inputs, each of which were richly interconnected by weighed connection lines. The output of this classifier was the prediction of prostate cancer (between 0 and 1).

\subsection{Statistical analysis}

A leave-one-ROI-out cross-validation approach [25] was used to separate the dataset into a training set and a test set. With this method, each ROI was used to test the classifier one time, while the others were used to train the classifier. This procedure was repeated until all of the 426 ROIs had been tested. Receiver operating characteristic (ROC) curve analysis was performed to evaluate the performance of this 
Table 3 The parameters of this devised ANN classifier structure

\begin{tabular}{ll}
\hline Parameters & Properties \\
\hline Training algorithm & Back-propagation (BP) \\
Layers & 3 \\
Number of neurons in the input layer & 5 \\
Number of neurons in the hidden layer & 14 \\
Number of neurons in the output layer & 1 \\
Learning rate & 0.1 \\
Epoch number & 500 \\
Learning algorithm & Levenberg-Marquardt (LM) \\
Transfer function & Logarithmic-sigmoid (Logsig) \\
\hline
\end{tabular}

CAD system [26-28], and two radiologists' scores were used for comparison. Areas under the ROC curves (AUC), sensitivity, specificity, positive predictive values (PPV), negative predictive values (NPV), and accuracy were utilised to evaluate the performance of this CAD system. All statistical tests were two sided, and $P<0.01$ was considered to indicate significant difference.

\section{Results}

Among the 71 patients recruited in our study, 35 (mean age $68.8 \pm 8.9$ years, range 40-82 years; tPSA $60.5 \pm 77.8 \mathrm{ng}$ $\mathrm{mL}^{-1}$, range $5.6-297.3 \mathrm{ng} \mathrm{mL}{ }^{-1}$ ) were confirmed to have PCa by biopsy, while the other 36 patients (mean age $67.8 \pm 6.4$ years, range 53-79 years; tPSA $11.7 \pm 8.1 \mathrm{ng} \mathrm{mL}^{-1}$, range $0.85-37.1 \mathrm{ng} \mathrm{mL}^{-1}$ ) were not detected of PCa by serial biopsy and long-term follow-up.

The performance of features extracted from T2WI is shown in Table 4. Most of the features (10/12) had significant difference $(P<0.01)$ between $\mathrm{PCa}$ and non-PCa in the $\mathrm{PZ}$, while only five features (sum average, minimum value,
SD, 10th percentile, and entropy) had significant difference in CG. These five features had significant difference in both PZ and CG. The importance of different images' features in PZ and CG is shown in Figure 3.

After SFS selection, six features (sum average, minimum value, SD, 10th percentile, contrast, and correction) remained as the inputs of the ANN classifier for PZ, and five features (sum average, minimum value, SD, 10th percentile, and entropy) were selected as the inputs of the ANN classifier for $\mathrm{CG}$.

When comparing the performance of any selected features alone, the sum average has the highest AUC and best accuracy in both PZ (AUC $=0.831 \pm 0.029$, accuracy of 0.794) and CG (AUC=0.718 \pm 0.044 , accuracy of 0.755). After combining the selected features by the devised ANN classifier, the AUC was $0.849 \pm 0.028$ (95\%, CI=0.796-0.903) for the prediction of the CAD system in PZ, which was smaller than that of the T2WI scores $(0.898 \pm 0.023(95 \%$, $\mathrm{CI}=0.852-0.943)$ ). AUC of the prediction of the CAD system in $\mathrm{CG}$ (Figure 4B, AUC $=0.821 \pm 0.038$ (95\%, CI= 0.746-0.895)) was also smaller than that of the T2WI scores $(0.857 \pm 0.035(95 \%, \mathrm{CI}=0.789-0.925))$. By selecting appropriate cut-offs ( 0.688 for PZ, 0.725 for CG), the CAD prediction can reach high accuracy and specificity while maintaining acceptable sensitivity ( 0.652 for PZ, 0.654 for $\mathrm{CG})$. The summary of the CAD performance and T2WI scores is shown in Table 5.

\section{Discussion}

This study made an indepth research into the prostate T2WI by a CAD system, which can differentiate malignancies from benign regions with an AUC of 0.849 in PZ and 0.821 in CG. There is already a consensus that multi-parameter

Table 4 The means and SDs of features extracted from T2-weighted images in PZ and CG

\begin{tabular}{|c|c|c|c|c|c|c|}
\hline \multirow{2}{*}{ Feature } & \multicolumn{3}{|c|}{ Peripheral zone } & \multicolumn{3}{|c|}{ Central gland } \\
\hline & $\mathrm{PCa}$ & Non-PCa & $P$-value & $\mathrm{PCa}$ & Non-PCa & $P$-value \\
\hline Sum average & $370.6 \pm 160.5$ & $661.5 \pm 244.5$ & $<0.01$ & $355.5 \pm 112.0$ & $463.4 \pm 154.8$ & $<0.01$ \\
\hline Minimum value & $298.6 \pm 142.8$ & $508.5 \pm 216.5$ & $<0.01$ & $289.3 \pm 89.88$ & $373.6 \pm 123.6$ & $<0.01$ \\
\hline Standard deviation & $35.74 \pm 15.56$ & $68.14 \pm 34.79$ & $<0.01$ & $33.85 \pm 16.81$ & $46.37 \pm 21.65$ & $<0.01$ \\
\hline Coefficient of variation & $11.18 \pm 4.30$ & $11.19 \pm 4.98$ & 0.982 & $11.84 \pm 4.350$ & $11.04 \pm 3.513$ & 0.194 \\
\hline 10th percentile & $323.9 \pm 148.5$ & $570.2 \pm 227.8$ & $<0.01$ & $313.4 \pm 98.05$ & $404.9 \pm 135.1$ & $<0.01$ \\
\hline Skewness & $0.126 \pm 0.509$ & $-0.189 \pm 0.697$ & $<0.01$ & $0.256 \pm 0.447$ & $0.212 \pm 0.591$ & 0.627 \\
\hline Kurtosis & $2.736 \pm 0.699$ & $3.019 \pm 1.366$ & 0.067 & $2.742 \pm 0.812$ & $2.912 \pm 1.045$ & 0.291 \\
\hline Contrast & $1.077 \pm 0.439$ & $0.842 \pm 0.379$ & $<0.01$ & $1.351 \pm 0.517$ & $1.292 \pm 0.545$ & 0.496 \\
\hline Correlation & $0.810 \pm 0.096$ & $0.859 \pm 0.072$ & $<0.01$ & $0.770 \pm 0.088$ & $0.776 \pm 0.098$ & 0.683 \\
\hline Energy & $0.075 \pm 0.022$ & $0.087 \pm 0.039$ & $<0.01$ & $0.065 \pm 0.018$ & $0.072 \pm 0.023$ & 0.076 \\
\hline Homogeneity & $0.675 \pm 0.060$ & $0.711 \pm 0.068$ & $<0.01$ & $0.637 \pm 0.060$ & $0.651 \pm 0.063$ & 0.154 \\
\hline Entropy & $5.454 \pm 0.248$ & $5.615 \pm 0.161$ & $<0.01$ & $5.446 \pm 0.229$ & $5.535 \pm 0.200$ & $<0.01$ \\
\hline
\end{tabular}

a) $* P$ values calculated by two-sample $t$-test were used to indicate the significance of the difference between features obtained in normal ROIs and cancerous ROIs. Data are mean \pm SD. 

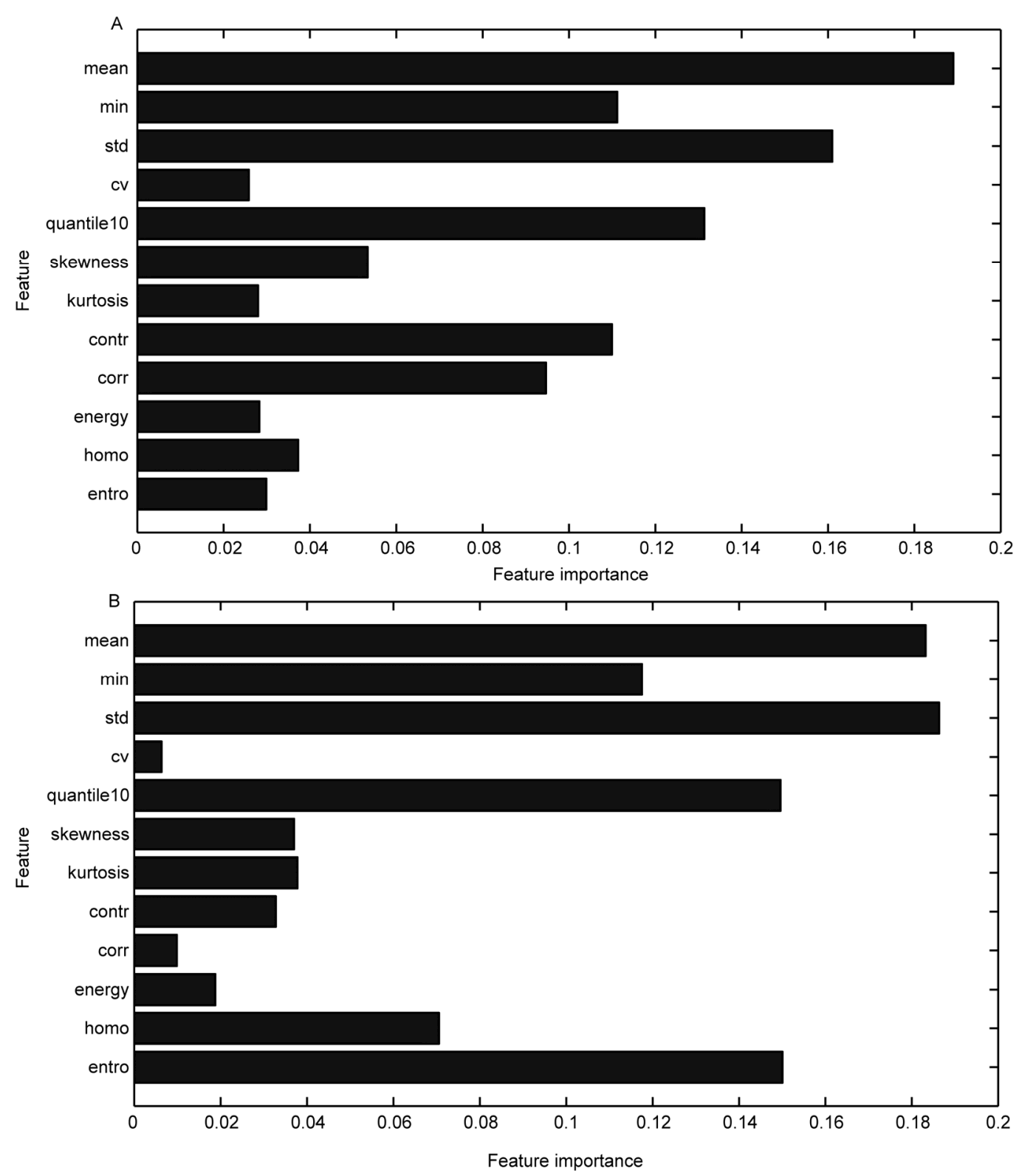

Figure 3 Importance of different images features in PZ (A) and CG (B). Mean, mean value; min, minimum value; std, standard deviation; cv, coefficient of variation; quantile 10, 10th percentile; contr, contrast; corr, correlation; homo, homogeneity; entro, entropy.

Table 5 Summary of the CAD performance of different groups ${ }^{\text {a) }}$

\begin{tabular}{ccccccccc}
\hline \multirow{2}{*}{ Position } & Subject & AUC $($ mean \pm std $(95 \%$, CI) $)$ & Cut-off & Sensitivity & Specificity & PPV & NPV & Accuracy \\
\hline \multirow{2}{*}{ PZ } & CAD prediction & $0.849 \pm 0.028(0.796-0.903)$ & 0.688 & 0.652 & 0.897 & 0.800 & 0.804 & 0.803 \\
& T2WI score & $0.898 \pm 0.023(0.852-0.943)$ & 3.000 & 0.728 & 0.979 & 0.957 & 0.851 & 0.882 \\
\hline \multirow{2}{*}{ CG } & CAD prediction & $0.821 \pm 0.038(0.746-0.895)$ & 0.725 & 0.654 & 0.912 & 0.739 & 0.873 & 0.840 \\
& T2WI score & $0.857 \pm 0.035(0.789-0.925)$ & 3.000 & 0.596 & 0.941 & 0.795 & 0.859 & 0.846 \\
\hline
\end{tabular}

a) AUC, area under the curve; $\mathrm{CI}$, confidence interval.

diagnosis should be used [5,22] in prostate MR imaging. As far as we know, few papers have been published that focused on the basic prostate T2-weighted MR imaging by using $\mathrm{CAD}$ and introducing the image features in detail with corresponding clinical implications.
Quantitative features extraction is the major difficulty affecting image processing and analysis. In this study, 12 quantitative features were extracted from T2WI. Humans have limited ability to discover and deal with the complex relationship of so many features. ANNs are artificial intel- 

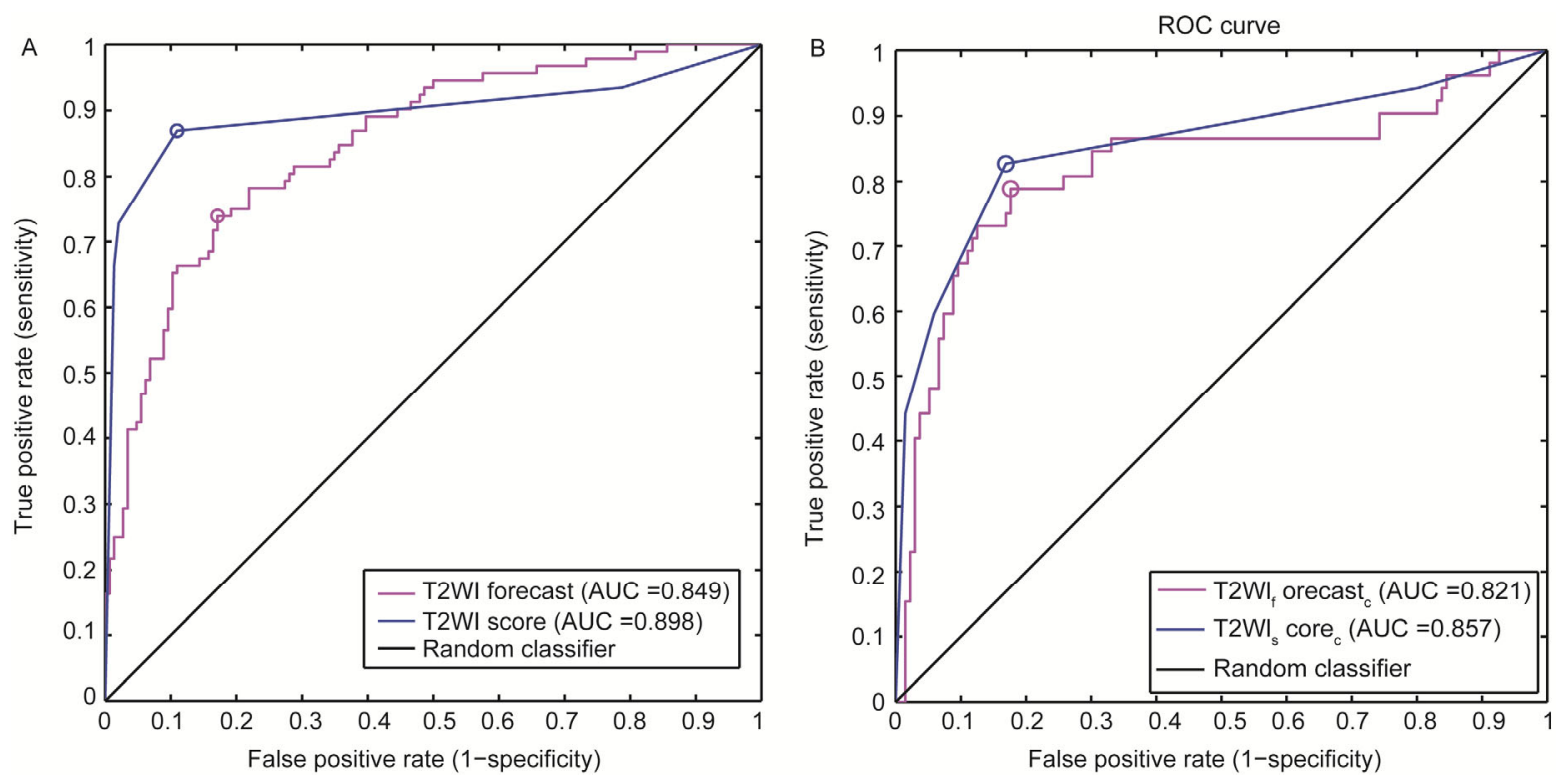

Figure 4 ROC curves for ROIs located in PZ (A) and CG (B). The purple curves stand for the performance of this CAD system and the blue curves indicate the results of the T2WI scores.

ligent systems that acquire knowledge through a learning process and use the knowledge to predict results from unseen information, like the processing of the human brain. Therefore, it has the potential capabilities to find the complex interactions between the input variables and the outcome.

Viswanath et al. [29] indicated that $\mathrm{CG}$ and $\mathrm{PZ}$ prostate cancers and non-cancers have significantly different quantitative and texture features on T2-weighted MR images. Detection of PCa by MR is most effective for tumours located in PZ, as an area of low signal intensity can be easily differentiated from high-signal-intensity normal tissue. Tumours in CG should be carefully sought, especially in the absence of a dominant tumour in PZ with increased PSA levels. In $\mathrm{CG}$, hyperplastic nodules make the signal more complicated than that of PZ, and low signal intensity of PCa in T2WI is easily confused with hyperplastic nodules. A homogeneous low-signal-intensity region in $\mathrm{CG}$ is a very important finding that supports the diagnosis of a transition zone tumour [30]. Therefore, the characteristics of CG and PZ were separately extracted and analysed in our research. After selective optimisation of 12 features extracted from T2WI, the features were verified as valid to apply in computer-aided PCa diagnosis. In PZ, 10 of the 13 features could distinguish PCa from non-PCa. But for CG, the number was only 5 .

The first three indicators of general features of both CG and PZ showed significant difference, and the coefficient of variation showed no obvious difference. For the sum average and the minimum, PCa both in CG and PZ appears as an area of low signal intensity in T2WI. It is easily differentiated from high-signal-intensity normal PZ tissue, but often with some difficulty in CG regions. The minimum cannot be easily detected by the human eyes, especially in the hyperplasia region of CG. This is one of the advantages of CAD. The low signal intensity is always homogeneous in the cancer region, both in CG and PZ [31], which means the standard deviation in the cancer region is lower than that of non-PCa.

The 10th percentile from the histogram showed an obvious difference between PCa and non-PCa, both in CG and $\mathrm{PZ}$. As the cancer is always a homogeneous low signal in T2WI, the distribution of pixels in the cancerous region must be skewed.

All the five features derived from the co-occurrence matrix were significantly different between $\mathrm{PCa}$ and non-PCa in $\mathrm{PZ}$, but only entropy was significantly different between $\mathrm{PCa}$ and non-PCa in CG. The underlying mechanism could be that the healthy prostate PZ consists of complicated tissue structures with normal glandular morphology, whose complex architectures contribute to higher roughness features on the T2-weighted MR images. The presence of PCa increases cellular density and changes microvasculature. As a result, the normal pattern is disrupted and the complexity of the ROI on the T2WI is reduced. Such a behaviour is also evident in Table 4, where relatively higher energy and entropy can be found in cancerous ROIs compared with those of non-cancer ROIs. As a result, the texture features reflect the complexities at different levels for those cancerous patterns.

For PZ, sum average contributed the largest part in distinguishing PCa, while SD became the most important feature for CG. The result is also consistent with our clinical experience. $\mathrm{PCa}$ in $\mathrm{PZ}$ appears as an area of low signal intensity that is always easy to differentiate from highsignal-intensity normal tissue. For CG, homogeneity of the 
low-signal-intensity region is a very important feature supporting the diagnosis of PCa. It is worth noting that one of the texture features "entropy" ranked in third place. This means the lesions in CG with features of homogeniety, low signal intensity, and a disorganised state have a higher possibility of cancer.

After combining the selected features by the devised ANN classifier, better results were achieved than by any feature alone. For PZ, the CAD system distinguished benign from malignant lesions with an AUC of 0.849 (95\% CI $=0.796-0.903)$, while the AUC for CG was 0.821 (95\% $\mathrm{CI}=0.746-0.895)$. The results were not worse than previous studies using multi-parameter MR including diffusion weighted (DWI) and/or dynamic contrast-enhanced MRI (DCE). In the studies combining T2WI, DWI, and DCE images, values of 0.79 and 0.83 for overall AUC were achieved [24,32]. The results were worse than the results of our study, which only used T2WI. The relatively small number of patients may be the cause of the lower diagnosis accuracy. Hambrock et al. [33] got better AUC values of $0.92(95 \% \quad \mathrm{CI}=0.88-0.96)$ for $\mathrm{PZ}$ and $0.87(95 \%$ $\mathrm{CI}=0.78-0.96)$ for $\mathrm{CG}$.

When compared with radiologists' scores, the prediction results of CAD were a little lower, but the prediction accuracy was more than $80 \%$, which was at an acceptable level. Because the two radiologists had 7 and 9 years of prostate MR diagnosis experience, they are very experienced. At present, the most important clinical value of the CAD system is to assist radiologists in making decisions by providing an objective chance of malignancy for any suspicious lesion with high reliability and repeatability, especially for less-experienced doctors. This is especially important for those patients with a low risk of $\mathrm{PCa}$ and in no need of a biopsy.

Time is an important issue for any techniques to be actually used in clinical practice. Many promising techniques have not been incorporated into clinical practice because they were time-consuming. This CAD system is time-saving and very efficient. Once the CAD system is ready, only a few seconds are needed for the result prediction, which is very convenient in clinical practice. Thus, diagnoses can be made more quickly and reliably.

There are several limitations in our research. First, we aimed to compare the diagnosis performance of a feature set by this CAD system, though this was not the right way to distinguish prostate malignancy from a benign region only using T2WI. Second, ROI and pathology were not strictly correlated, although they were identified by two experienced radiologists. Third, numbers of ROIs of cancer and non-cancer were not identical, so this may have resulted in a difference in the learning process. More ROIs will be used to train the ANN in later research in order to increase accuracy.

In conclusion, with computer-aided analysis of T2WI, many characteristic features with different diagnostic capabilities can be extracted. The outcome is convictive and helpful in medical diagnosis.

The authors declare that there is no conflict of interest.

This retrospective study was approved by the Hospital Medical and Ethical Review Committee (2010[224]). All patients or legal representatives signed informed consent. All patient records, information, and images were anonymised and de-identified prior to analysis. All procedures performed in studies involving human participants were in accordance with the ethical standards of the institutional and/or national research committee and with the 1964 Helsinki declaration and its later amendments or comparable ethical standards.

1 Siegel R, Naishadham D, Jemal A. Cancer statistics, 2012. CA Cancer J Clin, 2012, 62: 10-29

2 Cancer Registration Committee of the Japanese Urological Association. Clinicopathological statistics on registered prostate cancer patients in Japan: 2000 report from the Japanese Urological Association. Int J Urol, 2005, 12: 46-61

3 Quinn M, Babb P. Patterns and trends in prostate cancer incidence, survival, prevalence and mortality. Part I: international comparisons. BJU Int, 2002, 90: 162-173

4 Quinn M, Babb P. Patterns and trends in prostate cancer incidence, survival, prevalence and mortality. Part II: individual countries. BJU Int, 2002, 90: 174-184

5 Hoeks CM, Barentsz JO, Hambrock T, Yakar D, Somford DM, Heijmink SW, Scheenen TW, Vos PC, Huisman H, van Oort IM, Witjes JA, Heerschap A, Futterer JJ. Prostate cancer: multiparametric MR imaging for detection, localization, and staging. Radiology, 2011, 261: 46-66

6 Ahmed HU, Kirkham A, Arya M, Illing R, Freeman A, Allen C, Emberton M. Is it time to consider a role for MRI before prostate biopsy? Na Rev Clin Oncol, 2009, 6: 197-206

7 Kirkham AP, Emberton M, Allen C. How good is MRI at detecting and characterising cancer within the prostate? Eur Urol, 2006, 50: 1163-1174; discussion 1175

8 Sciarra A, Barentsz J, Bjartell A, Eastham J, Hricak H, Panebianco V, Witjes JA. Advances in magnetic resonance imaging: how they are changing the management of prostate cancer. Eur Urol, 2011, 59: 962-977

9 Bezzi M, Kressel HY, Allen KS, Schiebler ML, Altman HG, Wein AJ, Pollack HM. Prostatic carcinoma: staging with MR imaging at 1.5 T. Radiology, 1988, 169: 339-346

10 Schnall MD, Imai Y, Tomaszewski J, Pollack HM, Lenkinski RE, Kressel HY. Prostate cancer: local staging with endorectal surface coil MR imaging. Radiology, 1991, 178: 797-802

11 Cheikh AB, Girouin N, Colombel M, Marechal JM, Gelet A, Bissery A, Rabilloud M, Lyonnet D, Rouviere O. Evaluation of T2-weighted and dynamic contrast-enhanced MRI in localizing prostate cancer before repeat biopsy. Eur Radiol, 2009, 19: 770-778

12 Bloch BN, Furman-Haran E, Helbich TH, Lenkinski RE, Degani H, Kratzik C, Susani M, Haitel A, Jaromi S, Ngo L, Rofsky NM. Prostate cancer: accurate determination of extracapsular extension with high-spatial-resolution dynamic contrast-enhanced and T2weighted MR imaging-initial results. Radiology, 2007, 245: 176-185

13 Hricak H, Choyke PL, Eberhardt SC, Leibel SA, Scardino PT. Imaging prostate cancer: a multidisciplinary perspective. Radiology, 2007, 243: 28-53

14 Kundra V, Silverman PM, Matin SF, Choi H. Imaging in oncology from the University of Texas M. D. Anderson Cancer Center: diagnosis, staging, and surveillance of prostate cancer. AJR, 2007, 189: 830-844 
15 May F, Treumann T, Dettmar P, Hartung R, Breul J. Limited value of endorectal magnetic resonance imaging and transrectal ultrasonography in the staging of clinically localized prostate cancer. BJU Int, 2001, 87: 66-69

16 Lim HK, Kim JK, Kim KA, Cho KS. Prostate cancer: apparent diffusion coefficient map with T2-weighted images for detection-a multireader study. Radiology, 2009, 250: 145-151

17 Regge D, Halligan S. CAD: how it works, how to use it, performance. Eur J Radiol, 2013, 82: 1171-1176

18 Seltzer SE, Getty DJ, Tempany CM, Pickett RM, Schnall MD, McNeil BJ, Swets JA. Staging prostate cancer with MR imaging: a combined radiologist-computer system. Radiology, 1997, 202: 219-226

19 Chen W, Giger ML, Li H, Bick U, Newstead GM. Volumetric texture analysis of breast lesions on contrast-enhanced magnetic resonance images. Magn Reson Med, 2007, 58: 562-571

20 Li Q, Li F, Suzuki K, Shiraishi J, Abe H, Engelmann R, Nie Y, MacMahon H, Doi K. Computer-aided diagnosis in thoracic CT. Semin Ultrasound CT MR, 2005, 26: 357-363

21 Nappi JJ, Kim SH, Yoshida H. Volumetric detection of colorectal lesions for noncathartic dual-energy computed tomographic colonography. In: Proceedings of Annual International Conference of the IEEE Engineering in Medicine and Biology Society, 2012. 3740-3743

22 Barentsz JO, Richenberg J, Clements R, Choyke P, Verma S, Villeirs G, Rouviere O, Logager V, Futterer JJ, European Society of Urogenital R. ESUR prostate MR guidelines 2012. Eur Radiol, 2012, 22: 746-757

23 Madabhushi A, Udupa JK. Interplay between intensity standardization and inhomogeneity correction in MR image processing. IEEE Trans Med Imag, 2005, 24: 561-576

24 Niaf E, Rouviere O, Mege-Lechevallier F, Bratan F, Lartizien C. Computer-aided diagnosis of prostate cancer in the peripheral zone using multiparametric MRI. Phys Med Biol, 2012, 57: 3833-3851
25 Vos PC, Hambrock T, Hulsbergen-van de Kaa CA, Futterer JJ, Barentsz JO, Huisman HJ. Computerized analysis of prostate lesions in the peripheral zone using dynamic contrast enhanced MRI. Med Phys, 2008, 35: 888-899

26 Metz CE. ROC methodology in radiologic imaging. Invest Radiol, 1986, 21: 720-733

27 Metz CE, Herman BA, Shen JH. Maximum likelihood estimation of receiver operating characteristic (ROC) curves from continuouslydistributed data. Stat Med, 1998, 17: 1033-1053

28 Metz CE, Herman BA, Roe CA. Statistical comparison of two ROC-curve estimates obtained from partially-paired datasets. Med Decision Making, 1998, 18: 110-121

29 Viswanath SE, Bloch NB, Chappelow JC, Toth R, Rofsky NM, Genega EM, Lenkinski RE, Madabhushi A. Central gland and peripheral zone prostate tumors have significantly different quantitative imaging signatures on 3 Tesla endorectal, in vivo T2-weighted MR imagery. JMRI, 2012, 36: 213-224

30 Akin O, Sala E, Moskowitz CS, Kuroiwa K, Ishill NM, Pucar D, Scardino PT, Hricak H. Transition zone prostate cancers: features, detection, localization, and staging at endorectal MR imaging. Radiology, 2006, 239: 784-792

31 Boonsirikamchai P, Choi S, Frank SJ, Ma J, Elsayes KM, Kaur H, Choi H. MR imaging of prostate cancer in radiation oncology: what radiologists need to know. Radiographics, 2013, 33: 741-761

32 Ozer S, Langer DL, Liu X, Haider MA, van der Kwast TH, Evans AJ, Yang Y, Wernick MN, Yetik IS. Supervised and unsupervised methods for prostate cancer segmentation with multispectral MRI. Med Phys, 2010, 37: 1873-1883

33 Hambrock T, Vos PC, Hulsbergen-van de Kaa CA, Barentsz JO, Huisman HJ. Prostate cancer: computer-aided diagnosis with multiparametric 3-T MR imaging-effect on observer performance. Radiology, 2013, 266: 521-530

34 Haralick RM, Shanmuga K, Dinstein I. Textural features for image classification. Ieee T Syst Man Cyb, 1973, 23: 610-621

Open Access This article is distributed under the terms of the Creative Commons Attribution License which permits any use, distribution, and reproduction in any medium, provided the original author(s) and source are credited.

\section{Supporting Information}

The supporting information is available online at life.scichina.com and link.springer.com. The supporting materials are published as submitted, without typesetting or editing. The responsibility for scientific accuracy and content remains entirely with the authors. 